\title{
Severity in polycystic liver disease is associated with aetiology and female gender: Results of the International PLD Registry
}

\author{
René M. M. van Aerts ${ }^{1}$ | Wietske Kievit ${ }^{1}$ | Michiel E. de Jong ${ }^{1}$ | Curie Ahn ${ }^{2}$ | \\ Jesús M. Bañales $^{3}$ | Jana Reiterová ${ }^{4}$ | Frederik Nevens ${ }^{5}$ | Joost P. H. Drenth ${ }^{1}$ (D)
}

${ }^{1}$ Department of Gastroenterology and Hepatology, Radboud University Medical

Center, Nijmegen, The Netherlands

${ }^{2}$ Department of Internal Medicine, Seoul National University Hospital, Seoul, South Korea

${ }^{3}$ Department of Liver and Gastrointestinal Diseases, Biodonostia Research InstituteDonostia University Hospital, IKERBASQUE, CIBERehd, University of the Basque Country (UPV/EHU), San Sebastián, Spain

${ }^{4}$ Department of Nephrology, 1st Faculty of Medicine, Charles University and General University Hospital, Prague, Czech Republic

${ }^{5}$ Department of Gastroenterology and Hepatology, University Hospital KU Leuven, Leuven, Belgium

\section{Correspondence}

Joost P. H. Drenth, Department of Gastroenterology and Hepatology, Radboud University Medical Center, Nijmegen, The Netherlands.

Email: joostphdrenth@cs.com

Funding information

EASL Registry Research Grants Programme and unrestricted IPSEN grant for establishment of the PLD Registry. EASL and IPSEN had no role in the design or conduct of the study, or in the writing and submission of the manuscript. The Radboudumc Department of Gastroenterology and Hepatology is part of ERN-rare liver https://www.rare-liver.eu/.

Editor: Chun-Jen Liu

\begin{abstract}
Background \& Aims: Polycystic liver disease (PLD) occurs in two genetic disorders, autosomal-dominant polycystic kidney disease (ADPKD) and autosomal-dominant polycystic liver disease (ADPLD). The aim of this study is to compare disease severity between ADPKD and ADPLD by determining the association between diagnosis and height-adjusted total liver volume (hTLV).

Methods: We performed a cross-sectional analysis with hTLV as endpoint. Patients were identified from the International PLD Registry ( $>10$ liver cysts) and included in our analysis when PLD diagnosis was made prior to September 2017, hTLV was available before volume-reducing therapy (measured on computed tomography or magnetic resonance imaging) and when patients were tertiary referred. Data from the registry were retrieved for age, diagnosis (ADPKD or ADPLD), gender, height and hTLV.

Results: A total of 360 patients (ADPKD $n=241 ;$ ADPLD $n=119$ ) met our inclusion criteria. Female ADPKD patients had larger hTLV compared with ADPLD $(P=0.008)$. In a multivariate regression analysis, ADPKD and lower age at index CT were independently associated with larger hTLV in females, whereas in males a higher age was associated with larger hTLV. Young females ( $\leq 51$ years) had larger liver volumes compared with older females ( $>51$ years) in ADPKD.

Conclusion: Aetiology is presented as a new risk factor associated with PLD severity. Young females with ADPKD represent a subgroup of PLD patients with the most severe phenotype expressed in hTLV.
\end{abstract}

KEYWORDS

ADPKD, ADPLD, polycystic liver disease, risk factors

\section{1 | INTRODUCTION}

Autosomal-dominant polycystic kidney disease (ADPKD) is the most common genetic cause of end-stage kidney disease in adults.

Abbreviations: ADPKD, autosomal dominant polycystic kidney disease; ADPLD, autosomal dominant polycystic liver disease; CT, computed tomography; hTLV, height-adjusted total liver volume; MRI, magnetic resonance imaging; PLD, polycystic liver disease; PLD-Q, polycystic liver disease questionnaire; TLV, total liver volume.
Mutations in genes encoding polycystin 1 (PKD1) and polycystin 2 $(P K D 2)$ result in progressive renal cyst development and destruction of functional renal parenchyma. An important extra-renal manifestation of ADPKD is the presence of cysts in other organs such as the liver. Up to $94 \%$ of ADPKD patients over 35 years of age possess hepatic cysts. ${ }^{1}$ Liver cysts follow the onset of renal cysts and may result in polycystic liver disease (PLD), defined as the presence of more than 10 liver cysts. ${ }^{2}$ This clinical picture of ADPKD with PLD 
needs to be distinguished from autosomal-dominant polycystic liver disease (ADPLD), where patients develop PLD in the absence of a renal phenotype. In ADPLD, the genetic interaction network of endoplasmic reticulum resident proteins necessary for quality control and processing of glycoproteins is disrupted. ${ }^{3}$ PLD does not necessarily result in symptoms, but in patients who develop hepatomegaly abdominal discomfort, dyspnoea and early satiety cause a decreased quality of life and may lead to the development of sarcopenia. ${ }^{4}$

There is a broad range of PLD phenotypes, and studies have implicated age, female gender, and oestrogen use as risk factors for advanced disease. It is unknown whether patients with ADPKD or ADPLD have a similar risk of hepatomegaly. One study compared clinical features of ADPLD with that of ADPKD in a cohort of 53 patients. ${ }^{5}$ This study concluded that hepatic cysts were larger and more numerous in ADPLD, while symptoms resulting from hepatomegaly were similar. Total liver volume (TLV) as an endpoint was not investigated in this study. Better insight in phenotypical differences between ADPKD and ADPLD aid in the understanding of pathogenesis and natural course of disease and contributes to better counselling in clinical practice. As a result of the rarity of the disease and consequently lack of solid evidence, the recent establishment of an International PLD Registry provides us with the opportunity to explore clinical relevant questions in a large real-world cohort by collecting uniform clinical data from individual patients. ${ }^{6}$

The aim of this study is to compare disease severity between ADPKD and ADPLD by determining the association between diagnosis and height-adjusted total liver volume (hTLV).

\section{2 | MATERIALS AND METHODS}

\section{1 | Design and population International PLD Registry}

We made use of a recently established International PLD Registry that combines PLD cohorts from expert centres. Patients were included in the registry when referred to a hepatologist and diagnosed with either ADPLD with more than 10 liver cysts or ADPKD (based on the modified Ravine criteria) ${ }^{7}$ and PLD with more than 10 liver cysts proven by radiological imaging. Diagnosis of PLD was confirmed after inspection of radiologic images. In case of uncertainty, a second observer was asked to reassess the images and disagreement was resolved through discussion and mutual consent. Data collection for the registry was done retrospectively from the medical charts. The primary aim of this registry is to map the natural course of PLD and elucidate elements that affect disease progression. Data collection of PLD patients on a large scale provides us with the opportunity to answer research questions in selected cohorts and to select patients for clinical trials. Currently, over 1700 patients are included, and distributed over five centres (Radboud University Medical Center [Nijmegen, the Netherlands], University Hospitals Leuven [Leuven, Belgium], Seoul National University Hospital [Seoul, South-Korea], General University Hospital [Prague, Czech Republic] and Donostia University Hospital [San Sebastian, Spain]).

\section{Key points}

- In this study, we present the largest cohort of patients with polycystic liver disease (PLD) including both ADPKD (cysts in kidney and liver) and ADPLD (cysts in liver) patients. We show that PLD severity, expressed in liver volume, is highest in young female patients with ADPKD.

\subsection{Study design and population present analysis}

We performed a cross-sectional analysis comparing ADPKD and ADPLD with the focus on hTLV. For the present analyses, only centres who contributed both patients with ADPKD and ADPLD were selected to reduce potential selection bias. Patients were selected if (a) PLD diagnosis was made prior to September 2017, (b) liver volume data was available, as measured on computed tomography (CT) or magnetic resonance imaging (MRI) scan, and (c) tertiary referral. If more than one liver volume was available, first volume was selected. Patients from two tertiary centres could be included (Radboud University Medical Center [Nijmegen, the Netherlands] and University Hospital Leuven [Leuven, Belgium]). Both are tertiary referral centres for PLD and the registry contained both ADPKD and ADPLD patients. Other centres were excluded because no data on liver volume was available (Donostia University Hospital and General University Hospital Prague) and/or because imaging was only performed in ADPKD patients (General University Hospital Prague and Seoul National University Hospital). We excluded patients in whom first TLV was measured after any type of volume-reducing treatment (Figure 1). This was done because the main focus of our study was hepatomegaly as manifestation of the genetic diagnosis. For the current analysis, data were extracted from the registry database: age, gender and diagnosis (ADPKD/ADPLD), height and hTLV, and treatment.

\section{3 | Ethical considerations}

Concerning ethical approval of the PLD registry and thereby these analyses, formal evaluation was waived by the Institute Review Board Committee on Research Involving Human Subjects ArnhemNijmegen given the retrospective character of the data collection in the PLD Registry. The study was conducted in accordance with good clinical practice guidelines and the code of conduct for medical research (www.federa.org). No identifying patient data were collected, and all patient data were anonymously entered in the database.

\section{4 | Measurement of liver volume}

Total liver volume was measured by assessing CT or MRI scans using (semi-) automatic software as available in the participating centres. 


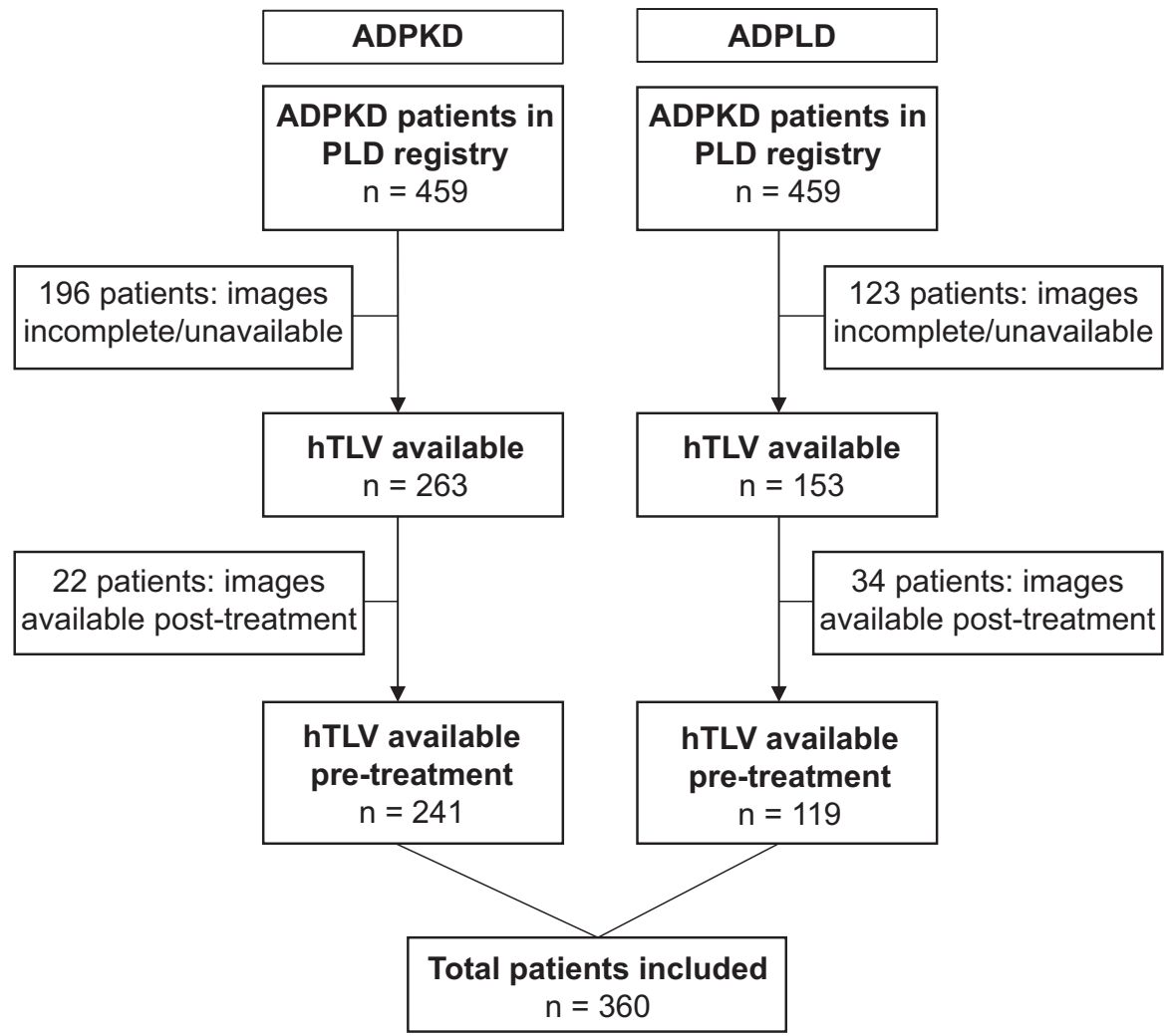

FIGURE 1 Study flow chart. A total of 360 patients were included (241 ADPKD, 119 ADPLD). ADPKD, autosomaldominant polycystic kidney disease; ADPLD, autosomal-dominant polycystic liver disease; hTLV, height-adjusted total liver volume; PLD, polycystic liver disease
In the Netherlands, TLV on CT was measured by 3D measurement of CT scan slices usingPinnacle 3 ${ }^{\circledR}$ version 8.0 (Philips, Eindhoven, The Netherlands). ${ }^{8,9}$ TLV measuring on MRI was performed with Analyze 11 software (AnalyzeDirect Inc, Overland Park, KS, USA) using a stereology method. ${ }^{10}$ In Belgium, TLV was measured with Volume software (Siemens, Erlangen, Germany). ${ }^{11,12}$ Specific details about each method have been prescribed previously. All imaging was performed as part of routine clinical care (eg, symptomatic disease, incidental finding, and familial screening) or in clinical trials. All liver volumes measured for trial purposes were measured blindly according to the study protocol. ${ }^{8-13}$ Liver volumetry for routine clinical care was performed by a trained physician (RvA). Previous studies have investigated inter-observer variability $(-0.2 \% \pm 2 \%)$ and agreement between techniques (mean difference $31 \mathrm{~mL}$ ). ${ }^{8,12}$

\section{5 | Classifications and definitions}

Patients were classified according to two separate classifications to provide better insight into the selection and distribution of patients between ADPKD and ADPLD. The Qian criteria grade disease severity from 0 to 4 depending on the number of cysts and the presence of symptoms. Grade 0; 0 cysts, Grade 1; 2-10 cysts (Grade 0 and 1 are not present in this cohort as corresponds to our inclusion criteria), Grade 2; 11-20 cysts, Grade 3; >20 cysts, Grade 4 ; >20 cysts and symptomatic hepatomegaly. ${ }^{14}$ Symptomatic disease was defined as having one or more symptoms in the PLD Questionnaire (PLD-Q) or the POLCA score, such as abdominal pain, early satiety and abdominal distension and likely to be associated with hepatomegaly. ${ }^{15,16}$ In addition we classified PLD as mild (hTLV $<1600 \mathrm{~mL} / \mathrm{m}$ ), moderate (hTLV $1600-3200 \mathrm{~mL} / \mathrm{m}$ ) and severe $(\mathrm{hTLV}>3200 \mathrm{~mL} / \mathrm{m}){ }^{4}$

\section{6 | Statistical analyses}

Descriptive statistical analyses were performed to quantify baseline characteristics expressed in mean (standard deviation [SD]) for normally distributed data, median (interquartile range [IQR]) for non-normally distributed data and proportions (\%) for categorical variables. To test for differences on those characteristics between ADPLD and ADPKD, we used independent $t$ test for normally distributed data, Mann-Whitney $U$ tests for non-normally distributed data and Fisher's exact test for categorical characteristics. TLV was divided by height in order to correct for differences in length. In case of missing height values ( $n=47$ ) we used the predicted height of a patient based on gender and year of birth according to Dutch National statistics database. ${ }^{17}$ hTLV was compared between patients with ADPKD and ADPLD. Several factors that are known to be associated with hTLV, such as age and gender, were considered as potential confounding factors. With these confounders, we performed univariate linear regression analysis with log-transformed hTLV as outcome. As evidence suggests that the disease behaviour with respect to liver volume are gender specific, we anticipated on this to incorporate a separate multivariable linear regression analysis for males and females with log-transformed hTLV as outcome and age (per decade) and diagnosis as fixed variable of primary interest". Log transformation was performed because of non-normal distribution 
TAB LE 1 Characteristics of the total study population and stratified for diagnosis

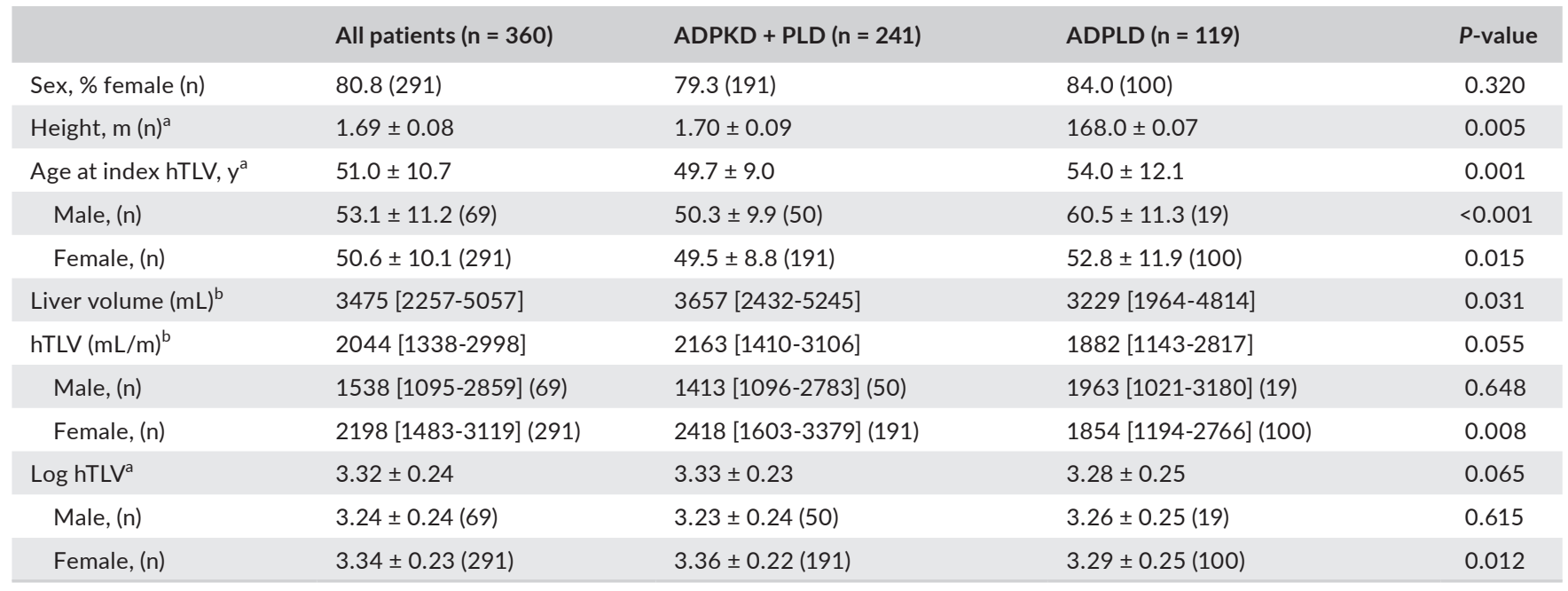

ADPKD, autosomal dominant polycystic kidney disease; ADPLD, autosomal-dominant polycystic liver disease; hTLV, height-adjusted total liver volume.

${ }^{a}$ Results given as mean \pm standard deviation.

${ }^{\mathrm{b}}$ Results given as median with interquartile range.
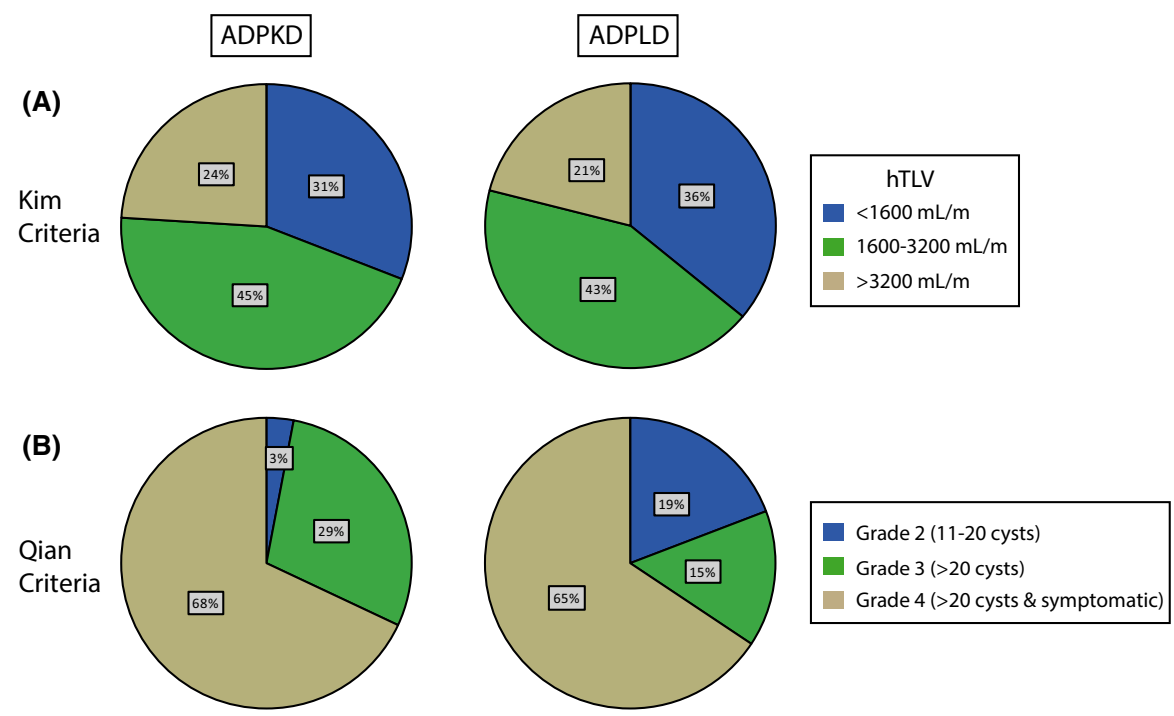

FIGURE 2 Distribution of ADPKD and ADPLD population based on two separate criteria (see Section 2). A, Kim criteria; no statistical differences in mild, moderate and severe hTLV, B, Qian Criteria; the proportion of symptomatic disease (grade 4) is evenly distributed between ADPKD and ADPLD, although the proportion of patients with less than 20 cysts is higher in ADPLD. hTLV, height-adjusted total liver volume; ADPKD, autosomal-dominant polycystic kidney disease; ADPLD, autosomal-dominant polycystic liver disease

of hTLV values. The beta-coefficient from the final model was back transformed in order to get an effect size which is easier to interpret.

\section{3 | RESULTS}

We included a total of 735 patients in the PLD registry from two tertiary centres in Belgium and the Netherlands. We selected 360 patients who met our inclusion criteria; 241 were diagnosed with ADPKD and 119 with ADPLD (Figure 1). Baseline characteristics are shown in Table 1. The majority of ADPKD (79.3\%) or ADPLD (84.0\%) patients were female. Male ADPLD patients were approximately 10 years older compared to male ADPKD patients at time of imaging $(P=<0.001)$. This difference in age was smaller in females (mean 3.3 years; $P=<0.015)$. ADPKD patients were taller than ADPLD which could be explained by the higher proportion of men in this group. The distribution in disease severity is shown in Figure 2.

Height-adjusted total liver volume was numerically larger in ADPKD than in ADPLD (2163 IQR 1410-3106 vs 1882 IQR 1143-2817, $P=0.055$ ), although this did not reach the threshold for statistical significance (Table 1). Gender contributed to the difference as in females hTLV was much larger in ADPKD than in ADPLD 
TAB LE 2 Univariate and multivariate regression model for associated risk factors

\begin{tabular}{|c|c|c|c|}
\hline Diagnosis (ADPKD/ADPLD) & & $-0.049(-0.10$ to 0.003$)$ & 0.065 \\
\hline Age at index hTLV (per decade) & & $-0.013(-0.036$ to 0.011$)$ & 0.288 \\
\hline & Multivariate & & \\
\hline & Beta $(95 \% \mathrm{Cl})$ & Effect size & $P$-value \\
\hline \multicolumn{4}{|l|}{ Male } \\
\hline Diagnosis (ADPKD/ADPLD) & $-0.040(-0.177$ to 0.098$)$ & $-8.8 \%(-33.5 \%$ to $25.3 \%)$ & 0.567 \\
\hline Age at index hTLV (per decade) & $-0.031(-0.058$ to -0.004$)$ & $-6.9 \%(-12.5 \%$ to $-0.9 \%)$ & 0.023 \\
\hline
\end{tabular}

ADPKD, autosomal dominant polycystic kidney disease; ADPLD, autosomal dominant polycystic liver disease; hTLV, height-adjusted total liver volume.

(2418 IQR 1603-3379 vs 1854 IQR 1194-2766, $P=0.008$; Figure

S1). These non-parametric findings were confirmed after performing parametric tests using Log hTLV as an outcome (Table 1).

In a univariate regression analysis of all PLD patients, female gender was associated with larger log hTLV $(P=0.003)$, whereas diagnosis and age at index hTLV failed to reach significance (Table 2). In females, multivariate regression analysis showed that diagnosis and age at index CT (per decade) were independently associated with hTLV. ADPLD patients had a $13.3 \%$ smaller hTLV compared to those with ADPKD ( $P=0.032$ ) and for each increased decade of age, hTLV decreased with $6.9 \%(P=0.023)$. In males, no association between diagnosis and hTLV was found. Of note, an opposite association was seen between hTLV and age at hTLV as liver volume increased with $17.8 \%$ per increased decade $(P=0.012)$ in males.

Because of these contradictory results between age and hTLV depending on gender, we decided to stratify for age and gender. We used the mean age at index hTLV as cut-off value. Young female PLD patients had larger liver volumes compared with older females (2485 IQR 1606-3639 vs 1925 IQR 1319-2718, $P=0.001$ ). This finding remained significant for ADPKD as diagnosis $(P=0.003)$ but not for ADPLD ( $P=0.406$; Figure 3$)$. In males, there was no significant difference between hTLV in younger and older men (1342 IQR 1095-2252 vs 1882 IQR 1076-3494, $P=0.113$ ), possibly because of lack of power (Figure S2).

During follow-up of patients in this cohort, several patients were in need for treatment. Median follow-up duration from index hTLV was 2.67 years (IQR 1.54-5.48) in ADPKD and 4.38 years (0.68-7.42) in ADPLD ( $P=0.189)$. In ADPLD aspiration sclerotherapy (30\%) and fenestration (11.8\%) were the most frequently used therapeutic options to reduce cyst and liver volume whereas in ADPKD these options were only used in $5 \%$ and $0.8 \%$, respectively. By contrast, liver transplantation was more frequently performed in ADPKD (13.3\%

\section{Females}

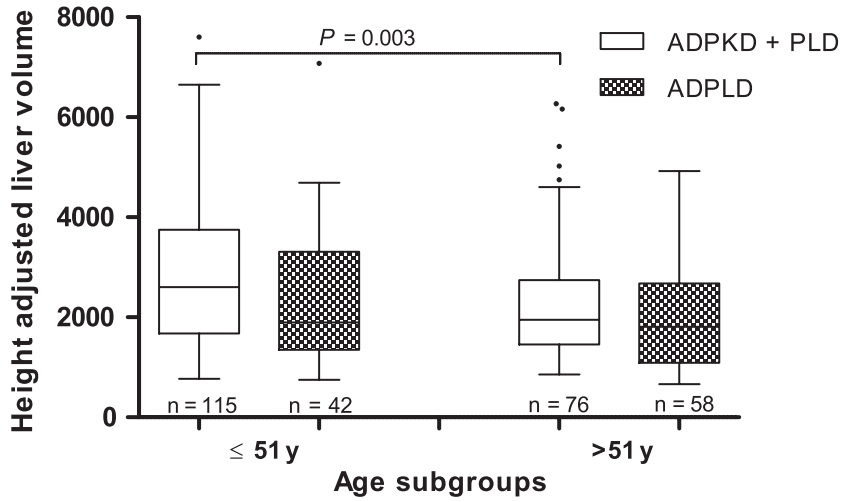

FIGURE 3 Whiskers Boxplot stratified for age and female gender. Younger female ADPKD patients have significant smaller hTLV compared with older ADPKD patients $(P=0.003)$. This was not significant in ADPLD $(P=0.406)$. Number of patients are displayed below each box. Whiskers Boxplot for males in Supporting Information. PLD, polycystic liver disease; ADPKD, autosomal-dominant polycystic kidney disease; ADPLD, autosomaldominant polycystic liver disease

of which $50 \%$ in combination with kidney transplantation) compared with ADPLD (5.0\%).

\section{4 | DISCUSSION}

The main finding of this study is that in patients tertiary referred to a hepatologist, females affected by ADPKD have larger hTLV compared to those with ADPLD, even after correction for age. In addition, we found that younger females with ADPKD ( $\leq 51$ years) have larger liver volumes compared with those older than 51 years of age. 
We compared disease severity with aetiological background to elucidate novel risk factors for PLD phenotype. The natural course of disease is difficult to predict, and it is difficult to counsel patients and discuss future perspectives in view of the lack of evidence. Until now, female gender is the only risk factor that is irrefutably associated with disease severity, again further confirmed in this study. ${ }^{18,19}$ For other risk factors such as age, oestrogen use and pregnancies, conflicting study results raise questions with respect to the true effect size on PLD severity. With the establishment of the International PLD Registry, we aim to collect a large population of PLD patients in order to clarify these uncertainties and answer new research questions for a better understanding of PLD. ${ }^{6}$

Previously, only one small cohort study surveying 19 ADPLD and 34 ADPKD patients, explored differences in PLD between the two diseases. This study focused on symptoms, complications and treatment. ${ }^{5}$ Its main finding was that the proportion of patients $>15$ liver cysts is higher in ADPLD population. This is in contrast with our study, where the proportion of patients with less than 20 cysts (Qian grade 2) is higher in ADPLD. These conflicting results may be subject to differences in patient selection and differences in counselling of ADPKD or ADPLD patients. Comparing symptoms between ADPKD and ADPLD shows the burden of underlying disease; however, it does not give a clear picture of PLD severity as patients with ADPKD might also suffer from enlarged kidneys leading to pressure-related symptoms. Our study provides to a more reliable comparison in PLD severity as our endpoint hTLV is focused on PLD only.

An important finding is that in female patients, liver volume is $6.9 \%$ smaller per decade of age. This contrasts with a recent study that suggested that hTLV increased with $10.0 \%$ per decade in female APDKD patient. ${ }^{20}$ These conflicting conclusions can be attributed to differences in disease populations. Our study is enriched with both ADPKD and ADPLD patients and covers a broad range of disease severity, whereas the study by Chebib studied mild ADPKD patients with index CT made at an earlier age. We hypothesize that age is associated with hTLV, though not in a linear pattern.

As is depicted in Figure 3, young females are more severely affected compared to their older counterparts. The mean age of patients in this cohort corresponds with the mean age at natural menopause based on literature ${ }^{21}$ suggesting that these finding and the differences with males may be explained by female hormonal status. In a controlled trial with 19 post-menopausal patients, exogenous use of oestrogen led to enlargement of the liver (+9\%) whereas liver size decreased in the control group $(-2 \%) .^{22}$ The association between oestrogen use and liver growth has not been confirmed in two recent cohort studies, ${ }^{20,23}$ possibly because of dose reduction in oestrogen in oral contraceptives in the last decades. ${ }^{24}$ Following the oestrogen hypothesis, liver volume would increase until the menopause and stabilize/decrease thereafter as oestrogen levels drop. As this cross-sectional study provides no individual data on natural growth, longitudinal studies are needed to confirm this hypothesis and to elucidate the association between oestrogen levels, menopause and liver growth.
The difference between older and younger females is only significant in ADPKD patients, possibly because of a lack of power in the ADPLD group. One could also speculate that the effect of oestrogen on cyst proliferation is different in ADPKD than in ADPLD. A previous study showed that oestrogens stimulate the proliferation of liver cyst derived epithelium by acting on specific oestrogen receptors in ADPKD patient. ${ }^{25}$ This interaction between oestrogens and other growth factors is presumed to play a key role in the PLD phenotype and its natural course in ADPKD.

A strength of our study is that we took hTLV as outcome to compare ADPKD and ADPLD. The size of the liver correlates with disease severity as patients with moderate to severe hepatomegaly have a 5 -fold higher risk of pressure-related complications. ${ }^{4}$ Quality of life decreases with severity of hepatomegaly, particularly in patients with $\mathrm{hTLV} \geq 1600 \mathrm{~mL} / \mathrm{m}^{26}{ }^{26}$ Therefore, $\mathrm{hTLV}$ serves as a reliable surrogate marker for PLD severity.

Another strength is that we draw conclusions in a homogenous cohort from tertiary centres. In our population, the proportion of patients who are symptomatic (Qian Grade 4) is equal between ADPKD and ADPLD, even as the distribution between mild, moderate and severe disease which supports the homogeneous composition of this cohort. Compared with other ADPKD cohorts in which the appearance of PLD is no inclusion criteria, the proportion of females in our ADPKD population is higher. ${ }^{20,23}$ This corresponds with the results of our study and previous literature as females are more at risk to develop severe hepatomegaly and therefore are more often tertiary referred because of symptomatic PLD compared with males.

This study comes with limitations. Only $49 \%$ of all patients in the registry could be included because they met the inclusion criteria of the present analyses. Excluded patients did not differ in gender and height compared with the included population (Table S1). The proportion of ADPLD was lower in the included group (33.1\%) compared with patients who did not meet the inclusion criteria $(41.9 \%$, $P=0.015)$ which suggest that radiological imaging (CT or MRI) is less performed in ADPLD. In the non-selected population, the proportion of ADPLD in Qian Grade 2 is $79.1 \%$ whereas the proportion in symptomatic patients (Grade 4) is higher in APDKD (58.5\%). Furthermore, we hypothesize that in mild ADPLD patients (hTLV $<1600 \mathrm{~mL} / \mathrm{m}$ ), ultrasound is sufficient for diagnosis and further imaging is waived. Therefore, we hypothesize that if hTLV would have been available for these patients, it probably would have further bolstered our findings that hTLV in ADPKD is larger. On the contrary, it is possible that mild ADPKD patients are managed by their nephrologist instead of referred to a tertiary centre which might lead to overestimation of liver volume in ADPKD. The main reason for imaging was unavailable for the majority of patients (57\%). In most cases, imaging was performed because of abdominal symptoms related to PLD, familial screening or incidental finding as imaging was performed for other purposes. Another limitation is that we were not able to analyze the role of exogenous oestrogen use and parity because of significant amount of missing data, and therefore low-quality data from our data sources. 
What is the clinical relevance of our findings? (a) This is the first study that shows that aetiology is associated with disease severity. It confirms and illustrates the high burden of PLD in ADPKD patients. A multi-specialist patient-centred approach is warranted in patients with ADPKD and PLD as advised by the European ADPKD Forum. ${ }^{27}$ Patients with symptomatic PLD or complications are preferable referred to an expert centre for further counselling and management. Our findings demonstrate the significance of PLD in a large cohort, especially in female ADPKD patients. (b) Based on our results, we provide evidence that in females' age is associated with PLD in a non-linear pattern. Younger females have smaller liver volumes which are in line with the hypothesis that liver growth in post-menopausal females is decreased because of lower oestrogen levels. It suggests that, if possible, a conservative management strategy should be priority in post-menopausal females. (c) For clinical practice, a prediction model to select patients at risk for severe hepatomegaly and predict the natural course of PLD based on age, gender, aetiology and hormonal status is warranted to offer better counselling and management advice. This study contributes to a future realization of this model. However first, longitudinal studies are needed to compare natural course of PLD between ADPKD and ADPLD and to determine the association between disease severity and female hormonal status.

\section{CONFLICT OF INTEREST}

The authors do not have any disclosures to report.

\section{ORCID}

Joost P. H. Drenth (iD http://orcid.org/0000-0001-8027-3073

\section{REFERENCES}

1. Bae KT, Zhu F, Chapman AB, et al. Magnetic resonance imaging evaluation of hepatic cysts in early autosomal-dominant polycystic kidney disease: the Consortium for Radiologic Imaging Studies of Polycystic Kidney Disease cohort. Clin J Am Soc Nephrol. 2006;1:64-69.

2. van Aerts R, van de Laarschot L, Banales JM, Drenth J. Clinical management of polycystic liver disease. J Hepatol. 2017; 68(4):827-837.

3. Wills ES, Roepman R, Drenth JP. Polycystic liver disease: ductal plate malformation and the primary cilium. Trends Mol Med. 2014;20:261-270.

4. Kim H, Park HC, Ryu H, et al. Clinical correlates of mass effect in autosomal dominant polycystic kidney disease. PLoS One. 2015;10:e0144526.

5. Hoevenaren IA, Wester R, Schrier RW, et al. Polycystic liver: clinical characteristics of patients with isolated polycystic liver disease compared with patients with polycystic liver and autosomal dominant polycystic kidney disease. Liver Int. 2008;28:264-270.

6. D'Agnolo HM, Kievit W, Andrade RJ, et al. Creating an effective clinical registry for rare diseases. United European Gastroenterol J. 2016;4:333-338.

7. Pei Y, Obaji J, Dupuis A, et al. Unified criteria for ultrasonographic diagnosis of ADPKD. J Am Soc Nephrol. 2009;20:205-212.
8. D'Agnolo HM, Kievit W, Takkenberg RB, et al. Ursodeoxycholic acid in advanced polycystic liver disease: A phase 2 multicenter randomized controlled trial. J Hepatol. 2016;65:601-607.

9. van Keimpema L, Nevens F, Vanslembrouck R, et al. Lanreotide reduces the volume of polycystic liver: a randomized, double-blind, placebo-controlled trial. Gastroenterology. 2009;137:1661-1668. e1-2.

10. Meijer E, Drenth JP, d'Agnolo $\mathrm{H}$, et al. Rationale and design of the DIPAK 1 study: a randomized controlled clinical trial assessing the efficacy of lanreotide to Halt disease progression in autosomal dominant polycystic kidney disease. Am J Kidney Dis. 2014;63(3):446-455.

11. Temmerman F, Ho TA, Vanslembrouck R, et al. Lanreotide reduces liver volume, but might not improve muscle wasting or weight loss, in patients with symptomatic polycystic liver disease. Clin Gastroenterol Hepatol. 2015;13:2353-2359.e1.

12. Temmerman F, Gevers T, Ho TA, et al. Safety and efficacy of different lanreotide doses in the treatment of polycystic liver disease: pooled analysis of individual patient data. Aliment Pharmacol Ther. 2013;38:397-406

13. Gevers T, Hol JC, Monshouwer R, et al. Effect of lanreotide on polycystic liver and kidneys in autosomal dominant polycystic kidney disease: an observational trial. Liver Int. 2015;35:1607-1614.

14. Qian Q, Li A, King BF, et al. Clinical profile of autosomal dominant polycystic liver disease. Hepatology. 2003;37:164-171.

15. Neijenhuis MK, Gevers TJ, Hogan MC, et al. Development and validation of a disease-specific questionnaire to assess patient-reported symptoms in polycystic liver disease. Hepatology. 2016;64:151-160.

16. Temmerman F, Dobbels F, Ho TA, et al. Development and validation of a polycystic liver disease complaint-specific assessment (POLCA). J Hepatol. 2014;61:1143-1150.

17. Statistiek CBvd. Lengte en gewicht van personen, ondergewicht en overgewicht; vanaf 1981. Volume 2016: CBS, 2016.

18. van Keimpema L, de Koning DB, van Hoek B, et al. Patients with isolated polycystic liver disease referred to liver centres: clinical characterization of 137 cases. Liver Int. 2010;31(1):92-98.

19. Gevers TJ, Inthout J, Caroli A, et al. Young women with polycystic liver disease respond best to somatostatin analogues: a pooled analysis of individual patient data. Gastroenterology. 2013;145:357-365.e1-2.

20. Chebib FT, Jung Y, Heyer CM, et al. Effect of genotype on the severity and volume progression of polycystic liver disease in autosomal dominant polycystic kidney disease. Nephrol Dial Transplant. 2016;31:952-960

21. Yarde F, Broekmans FJ, van der Pal-de Bruin KM, et al. Prenatal famine, birthweight, reproductive performance and age at menopause: the Dutch hunger winter families study. Hum Reprod. 2013:28:3328-3336.

22. Sherstha R, McKinley C, Russ P, et al. Postmenopausal estrogen therapy selectively stimulates hepatic enlargement in women with autosomal dominant polycystic kidney disease. Hepatology. 1997:26:1282-1286.

23. Hogan MC, Abebe K, Torres VE, et al. Liver involvement in early autosomal-dominant polycystic kidney disease. Clin Gastroenterol Hepatol. 2015;13:155-164.e6.

24. Maguire $\mathrm{K}$, Westhoff $\mathrm{C}$. The state of hormonal contraception today: established and emerging noncontraceptive health benefits. Am J Obstet Gynecol. 2011;205:S4-S8.

25. Alvaro D, Mancino MG, Onori $P$, et al. Estrogens and the pathophysiology of the biliary tree. World J Gastroenterol. 2006;12:3537-3545.

26. Neijenhuis MK, Kievit W, Verheesen SM, et al. Impact of liver volume on polycystic liver disease-related symptoms and quality of life. United European Gastroenterol J. 2018;6(1):81-88.

27. Harris T, Sandford R, de Coninck B, et al. European ADPKD Forum multidisciplinary position statement on autosomal dominant 
polycystic kidney disease care: European ADPKD Forum and Multispecialist Roundtable participants. Nephrol Dial Transplant. 2017;33(4):563-573.

\section{SUPPORTING INFORMATION}

Additional supporting information may be found online in the Supporting Information section at the end of the article.
How to cite this article: van Aerts RMM, Kievit W, de Jong $\mathrm{ME}$, et al. Severity in polycystic liver disease is associated with aetiology and female gender: Results of the International PLD Registry. Liver Int. 2018;00:1-8. https://doi. org/10.1111/liv.13965 